\title{
SEPARAÇÃO ENANTIOSSELETIVA DA OXIBUTININA: UMA INVESTIGAÇÃO TEÓRICA E EXPERIMENTAL
}

\author{
Lilian Christina Zinn, Camilla Fonseca Silva ${ }^{\mathrm{a}}$, Luciana Guimarães ${ }^{\mathrm{a}}$, Keyller Bastos Borges ${ }^{\mathrm{a}}$ e Clebio Soares Nascimento

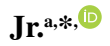 \\ a'Departamento de Ciências Naturais, Universidade Federal de São João del-Rei, Campus Dom Bosco, 36301-160 São João del- \\ Rei - MG, Brasil
}

Recebido em 04/05/2021; aceito em 10/08/2021; publicado na web em 27/08/2021

\begin{abstract}
ENANTIOSELECTIVE SEPARATION OF OXYBUTYNIN: A THEORETICAL AND EXPERIMENTAL INVESTIGATION. In this work, we have studied both experimentally and theoretically the oxybutynin chiral discrimination. According to the main results, the enantioseparation of oxybutynin was efficiently achieved by high performance liquid chromatography in normal phase using the Chiralpak ${ }^{\circledR} \mathrm{AD}$ column, which has amylose tris (3,5-dimethylphenylcarbamate) as a chiral selector. The energetic and structural parameters obtained via density functional theory calculations pointed out the chiral discrimination as well as the enantiomeric elution order of oxybutynin, thus explaining, at molecular level, the experimental data. Finally, the strength of the hydrogen bonds played a key role in the discrimination between the oxybutynin diastereomeric complexes formed.
\end{abstract}

Keywords: theoretical calculations, Density Functional Theory, chiral separation, diasteroisomeric complexes, oxybutynin.

\section{INTRODUÇÃo}

Problemas relacionados ao trato urinário são uma crescente preocupação em todo o mundo, sendo que as patologias mais comuns ${ }^{1}$ são a incontinência urinária (perda involuntária de urina) e a bexiga hiperativa, que é uma doença reconhecida pela urgência urinária, com aumento da frequência de micção. Essa urgência é de difícil controle e seus sintomas estão normalmente ligados à hiperatividade do músculo detrusor humano, no momento que este se contrai de forma inapropriada, durante o período de enchimento da bexiga. ${ }^{2}$

A acetilcolina é o neurotransmissor responsável pela contração do músculo detrusor. Fármacos da classe dos antimuscarínicos, também conhecidos como fármacos anticolinérgicos, são compostos sintéticos, conhecidos por sua capacidade de bloquear/inibir a atividade do receptor muscarínico da acetilcolina. ${ }^{3}$ Tais fármacos atuam reduzindo a atividade muscular espontânea, durante o período de enchimento da bexiga, diminuindo, consequentemente, a frequência urinária e intensidade de contração do músculo detrusor. ${ }^{4}$

Dentre os fármacos antimuscarínicos comercializados mundialmente, os quais têm mostrado eficiência em reduzir os principais sintomas, em até $75 \%$ dos casos, ${ }^{5}$ destaca-se a oxibutinina (OXI) (Figura 1(A)), um fármaco quiral, comercializado como racemato e na forma de cloridrato de oxibutina (nome químico: cloridrato de 4-dietilamino-2-butil-fenil-ciclohexil-glicolato), muito utilizado em função de ser o mais barato e mais eficiente dentre os demais, além de ser também disponível na forma de adesivos transdérmicos. A OXI também exerce efeito analgésico local na mucosa vesical e efeito antiespasmódico diretamente sobre o músculo liso, bem como inibe a ação muscarínica da acetilcolina sobre a musculatura lisa. ${ }^{6,7}$

A OXI, quando administrada por via oral, sofre pronunciada biotransformação hepática levando à formação da $N$-desetiloxibutinina (DEO) (Figura 1(B)), que também apresenta atividade anticolinérgica semelhante ao fármaco. ${ }^{8}$ Esse metabólito, junto com o fármaco, contribui para o efeito adverso de boca seca, o que leva muitos pacientes a desistirem do tratamento ou à necessidade de ajuste de dose. Outros efeitos colaterais adversos podem ser: taquicardia, constipação e visão turva. ${ }^{9}$ Nesse sentido, uma forma de minimizar tais efeitos sistêmicos

*e-mail: clebio@ufsj.edu.br é administrar uma formulação reduzindo a dose da OXI, porém, com aumento na concentração de DEO, ou ainda por meio de formulações orais de liberação controlada. ${ }^{10,11}$
(A)<smiles>CCN(CC)CC#CCOC(=O)[C@](O)(c1ccccc1)C1CCCCC1</smiles>

$(R)-\mathrm{OXI}$

(B)

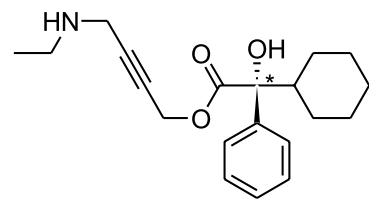

(R)-DEO<smiles>CCN(CC)CC#CCOC(=O)[C@](O)(c1ccccc1)C1CCCCC1</smiles>

(S)-OXI

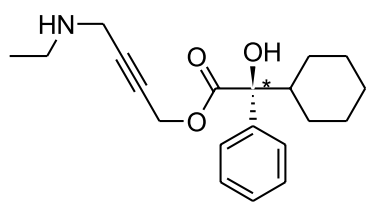

(S)-DEO
Figura 1. Estruturas químicas (A) dos enantiômeros das bases livres $(R)$-OXI e (S)-OXI e (B) (R)-DEO e (S)-DEO. O asterisco representa o centro estereogênico de cada molécula

A OXI e a DEO apresentam algumas similaridades entre si, além de suas estruturas químicas. Uma propriedade que se destaca é a presença de um centro estereogênico (carbono quiral), que confere à ambas as moléculas, a existência de um par de enantiômeros, conforme evidenciado na Figura 1. Dessa forma, interações estereoespecíficas de cada enantiômero com receptores biológicos promovem diferentes respostas, devido às diferentes propriedades farmacodinâmicas e farmacocinéticas dos mesmos. ${ }^{12-14}$

Embora se saiba que os $(R)$-enantiômeros da OXI e da DEO têm atividades antimuscarínicas superiores em relação aos $(S)$ enantiômeros, sendo que $(R)$-OXI e $(R)$-DEO são os enantiômeros responsáveis pela potência anticolinérgica, enquanto o $(S)$-OXI e $(S)$-DEO contribuem para uma menor incidência de efeitos adversos, ligando-se mais facilmente às glicoproteínas, ${ }^{14,15}$ quando administrados de forma separada, os enantiômeros não têm efeitos terapêuticos tão significativos, em comparação aos resultados alcançados quando ocorre a administração da mistura racêmica. ${ }^{15,16}$ 
Tais fatos tornam importante o desenvolvimento de técnicas e métodos analíticos com alto poder de separação de enantiômeros, conhecidos como métodos de separação enantiosseletiva, os quais permitem a identificação de cada enantiômero individualmente, visando um melhor entendimento acerca do fármaco em questão. ${ }^{17,18}$

Diferentes técnicas analíticas têm sido utilizadas corriqueiramente, de forma eficiente, na separação enantiosseletiva. Essas são normalmente divididas em dois grandes grupos principais: as técnicas de eletromigração - como a eletroforese capilar - e as técnicas cromatográficas - como a cromatografia gasosa e a cromatografia líquida de alta eficiência (HPLC, do inglês High Performance Liquid Chromatography ). ${ }^{19-21}$ Ao se empregar HPLC, para que cada enantiômero apresente uma interação distinta com as fases estacionária e móvel, uma estratégia recorrente diz respeito à utilização de um seletor quiral (SQ) na fase estacionária, a fim de possibilitar a formação dos chamados complexos diasteroisoméricos (CDTs). Tais complexos diferem entre si quanto ao arranjo estrutural e quanto à estabilidade energética, tendo em vista o estabelecimento de distintas interações intermoleculares dos enantiômeros com o SQ. ${ }^{22}$

Os tipos de SQs podem ser agrupados, basicamente, em três principais categorias que são mais utilizadas: $i$ ) derivados de polissacarídeos (principalmente amilose e celulose), ii) proteínas e iii) antibióticos macrocíclicos..$^{23} \mathrm{O}$ uso de colunas quirais derivadas de polissacarídeos é extenso, pois os mesmos podem ser convertidos em diferentes derivados - como triésteres e carbamatos - que geram novos sítios de reconhecimento quiral, proporcionando mais interações - como ligações de hidrogênio, dispersão de London, $\pi-\pi$ stacking - entre a fase estacionária e o analito, levando a velocidades de retenção e trajetórias distintas para cada um dos enantiômeros. ${ }^{24}$

Alebic-Kolbah \& Zavitsanos ${ }^{25}$ realizaram a análise enantiosseletiva da OXI em plasma empregando a coluna Chiralpak ${ }^{\circledR}$ AD no modo normal. A coluna quiral $\alpha_{1}$-glicoproteína ácida foi utilizada no modo normal por Zobrist e colaboradores ${ }^{15}$ e Mizushima ${ }^{14}$ para a resolução dos enantiômeros da OXI em plasma. Fonseca e colaboradores ${ }^{26}$ também desenvolveram a separação cromatográfica enantiosseletiva da OXI no modo normal para estudos de biotranformação in vitro empregando a coluna Chiralpak ${ }^{\circledR} \mathrm{AD}$.

Nesse contexto, a fim de compreender, em nível molecular, o processo de separação enantiosseletiva, auxiliando, dessa forma, a pesquisa experimental a elucidar a ordem de eluição enantiomérica (OEE), a Química Computacional tem se mostrado uma excelente ferramenta complementar para estudos envolvendo separação quiral. $^{27-29}$ Diversos estudos têm sido relatados na literatura visando analisar, em nível molecular, o processo de formação dos CDTs (enantiômeros + seletor quiral), além de obter estruturas e propriedades energéticas envolvidas em suas formações. Em tais estudos, a fase estacionária quiral baseada na amilose tris(3,5-dimetilfenilcarbamato) (ADMPC, do inglês amylose tris (-3,5-dimethylphenylcarbamate)) têm sido uma das mais aplicadas em simulações de dinâmica e docking molecular. ${ }^{27-29}$ Métodos quantomecânicos puros, a exemplo da Teoria do Funcional de Densidade (DFT, do inglês Density Functional Theory), têm sido ainda pouco usados, devido ao custo computacional elevado associado. ${ }^{30-32}$

Em trabalho recente, realizado em nosso grupo de pesquisa, Silva e colaboradores conduziram um estudo experimental/teórico visando a discriminação quiral do fármaco anti-helmíntico e antiparasitário, praziquantel (PZQ). Do ponto de vista teórico, parâmetros estruturais e energéticos, obtidos via cálculos DFT, apontaram a ordem de eluição dos enantiômeros do PZQ, cujos dados corroboraram perfeitamente com os dados experimentais obtidos. ${ }^{33}$

Diante do exposto, o objetivo do presente trabalho foi investigar, experimentalmente e teoricamente, via experimentos em HPLC e cálculos DFT, o mecanismo de separação enantiosseletiva da OXI, empregando a coluna Chiralpak ${ }^{\circledR}$ AD (seletor quiral, ADMPC), visando, fundamentalmente, a determinação da OEE. Cabe ressaltar que, até o momento, não foram encontrados relatos na literatura de estudos teóricos envolvendo a enantiosseparação da OXI, conferindo assim, ineditismo ao presente trabalho.

\section{MATERIAIS E MÉTODOS}

\section{Seção experimental}

\section{Materiais e reagentes}

Para análise por HPLC, os seguintes solventes orgânicos foram utilizados para a preparação de fases móveis e de soluções-padrão: $n$-hexano, metanol, etanol e isopropanol provenientes da J. T. Baker ${ }^{\circledR}$ Inc (Phillipsburg, EUA). Dietilamina foi utilizada no preparo da fase móvel também sendo também obtida da J. T. Baker ${ }^{\circledR}$ Inc (Phillipsburg, EUA). Empregou-se água purificada no sistema MILLI-Q PLUS ${ }^{\circledR}$ Millipore / Millipore Corporation (Bedford, EUA).

\section{Soluções-padrão}

O racemato de cloridrato de oxibutinina (99\%) foi gentilmente cedido por Watson Laboratories (Corona, CA, EUA). As soluções-padrão de oxibutinina foram preparadas em metanol nas concentrações de 1,0-400,0 $\mu \mathrm{g} \mathrm{mL}^{-1}$, a partir de uma solução estoque de $1,0 \mathrm{mg} \mathrm{mL}^{-1}$. As soluções preparadas foram estocadas a $-20^{\circ} \mathrm{C}$ e na ausência de luz, podendo ser usadas em até três dias sem sinais de degradação. ${ }^{26}$

\section{Equipamentos}

A análise enantiosseletiva da oxibutinina foi realizada em cromatógrafo Shimadzu ${ }^{\circledR}$ (Kyoto, Japão), composto por uma bomba modelo LC-10AT, um detector por absorção no UV-Visível modelo SPD-10Avp operando em $227 \mathrm{~nm}$ e um controlador SCL-10Avp. As injeções foram feitas manualmente em injetor Rheodyne ${ }^{\circledR}$ modelo 7125 (Cotati, USA) com amostrador de $20 \mu \mathrm{L}$. Os dados foram coletados e analisados pelo software Solutions Shimadzu ${ }^{\circledR}$ (Kyoto, Japão). A separação enantiosseletiva foi realizada em uma coluna quiral Chiralpak ${ }^{\circledR} \mathrm{AD}(250 \mathrm{~mm} \times 4,6 \mathrm{~mm}, 10 \mu \mathrm{m})$ em condições de fase normal.

\section{Seção teórica}

Todos os cálculos de otimização de geometria e frequências harmônicas foram realizados empregando-se a DFT, em nível M06-2X/6-31G(d,p) $)^{34}$ para os enantiômeros $(R)$ e $(S)$ da OXI, para o modelo reduzido da ADMPC, bem como, para os respectivos CDTs formados entre os enantiômeros e fase estacionária quiral. Todas as estruturas foram completamente otimizadas e caracterizadas como mínimos verdadeiros na Superfície de Energia Potencial (SEP) através da análise das frequências harmônicas, sendo todas as frequências calculadas reais. O funcional M06-2X foi escolhido por incluir em seu formalismo efeitos de dispersão, os quais são comumente observados em complexos moleculares, como os CDTs. ${ }^{35}$

O efeito do solvente foi considerado de forma implícita através do Modelo Contínuo de Solvatação baseado na Densidade (SMD, do inglês Solvation Model Density). ${ }^{36}$ Utilizou-se o $n$-hexano $(\varepsilon=1,88)$ uma vez que, experimentalmente, a fase móvel continha esse solvente em maior proporção (ver seção experimental).

As propriedades energéticas obtidas para formação dos CDTs - energia eletrônica $(\Delta \mathrm{E})$ e energia livre de Gibbs $(\Delta \mathrm{G})$ - foram calculadas a partir das Equações (1) e (2).

$$
\begin{gathered}
\Delta \mathrm{E}=\mathrm{E}_{\mathrm{CDT}}-\left(\mathrm{E}_{\mathrm{OXI}}+\mathrm{E}_{\mathrm{ADMPC}}\right) \\
\Delta \mathrm{G}=\mathrm{G}_{\mathrm{CDT}}-\left(\mathrm{G}_{\mathrm{OXI}}+\mathrm{G}_{\mathrm{ADMPC}}\right)
\end{gathered}
$$


Ao realizar cálculos de energia de complexação, envolvendo sistemas contendo interações não covalentes, utilizando o Modelo da Supermolécula ${ }^{37}$ surge um erro associado, chamado de erro de superposição de bases (BSSE, do inglês Basis Set Superposition Error), o que causa um abaixamento artificial da energia de estabilização do complexo molecular em relação às espécies interagentes isoladas. Nesse sentido, o BSSE foi avaliado utilizando a abordagem de Boys e Bernardi. ${ }^{38}$

Todos os cálculos foram realizados no pacote computacional Gaussian $09^{39}$ e os programas GaussView 5.0 e ArgusLab 4.0.1, utilizados como ferramentas gráficas para construção de modelos moleculares e visualização dos resultados de cálculo.

\section{RESULTADOS E DISCUSSÃO}

\section{Seção experimental}

A separação enantiosseletiva da OXI foi avaliada baseada em trabalho anterior, ${ }^{26}$ na qual empregou-se a coluna Chiralpak ${ }^{\circledR} \mathrm{AD}$ em condições de fase normal. Dessa forma, várias modificações da fase móvel foram realizadas para se adequar a uma melhor separação cromatográfica. Depois de alguns experimentos, variando as proporções de fase móvel e solventes orgânicos modificadores, verificou-se que $n$-hexano: isopropranol: etanol: dietilamina (94:4:2:0,05, $v / v / v / v)$ foi suficiente para promover uma separação de linha de base (Resolução, $R s>3$ ) dos enantiômeros. A Figura 2 apresenta a separação enantiosseletiva da OXI.

A ordem de eluição foi baseada em trabalhos anteriores, ${ }^{25,26}$ nos quais foram realizadas análises enantiosseletivas empregando soluções-padrão dos enantiômeros puros de $(R)$-OXI e $(S)$-OXI. Os enantiômeros da OXI foram coletados de acordo com as condições estabelecidas por Alebic-Kolbach \& Zavitsanos ${ }^{25}$ e a OEE posteriormente, foi confirmada por Fonseca e colaboradores. ${ }^{26}$ Após a coleta dos enantiômeros separados e já identificados, eles foram injetados na condição descrita na Figura 2. Como conclusão final, constatou-se que o $(R)$-enantiômero do fármaco elui mais rapidamente que seu antípoda $(S)$-enantiômero.

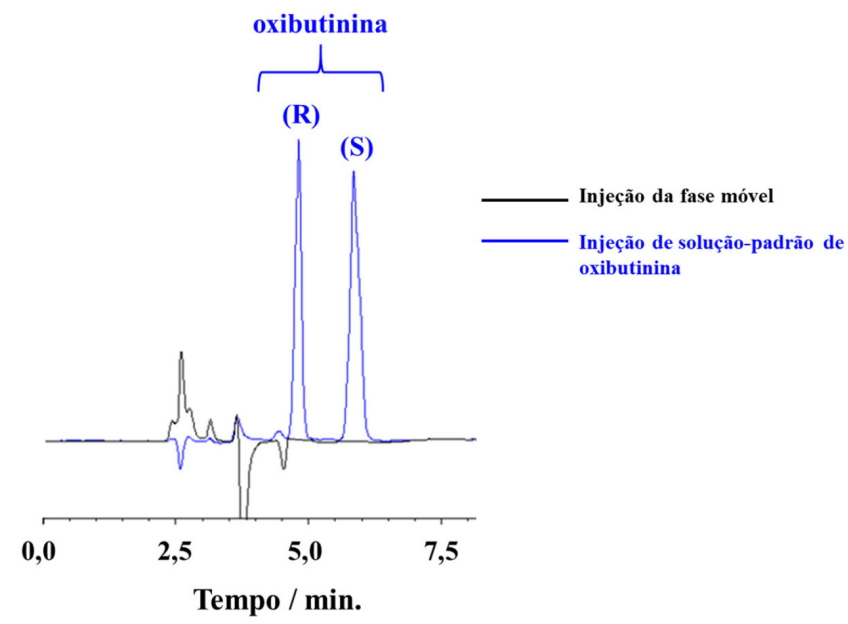

Figura 2. Cromatograma referente à separação enantiosseletiva da oxibutinina. Condições cromatográficas: coluna Chiralpak ${ }^{\circledR} A D(250 \mathrm{~mm} \times 4,6 \mathrm{~mm}$, $10 \mu \mathrm{m})$; fase móvel constituída por n-hexano: isopropranol: etanol: dietilamina (94: 4: 2: 0,05, v/v/v/v); vazão de $1 \mathrm{~mL} \mathrm{~min}^{-1}$ e detecção em $210 \mathrm{~nm}$. Em azul destaca-se a separação dos enantiômeros da OXI em solução-padrão e em preto a linha de base referente à fase móvel estudada

A ordem de eluição da OXI obtida ainda é confirmada por trabalhos encontrados na literatura. A exemplo, tem-se o trabalho de Ali e colaboradores ${ }^{40}$ em que, ao utilizar a CLAE com o mesmo seletor quiral e fase móvel bastante semelhante - sem o etanol e com proporções ligeiramente diferentes dos demais solventes - o enantiômero $(S)$ apresentou-se mais retido no sistema cromatográfico do que o $(R)$. Zhang e colaboradores ${ }^{41}$ também propuseram a mesma OEE pra $(S)$-OXI e $(R)$-OXI.

\section{Seção teórica}

Embora os modelos teóricos utilizados para descrição da estrutura dos SQs derivados de polissacarídeos sejam complexos, ou seja, contêm um grande número de átomos e com a estrutura helicoidal evidenciada (Figura 3(A)), diversos autores concordam que o mecanismo de discriminação quiral desta classe de SQs passam, majoritariamente, por interações dos analitos com os grupos amino $(-\mathrm{NH})$ e carbonila $(\mathrm{C}=\mathrm{O})$ da fase estacionária, além de possíveis interações $\pi-\pi$, no caso de racematos aromáticos e estéricas repulsivas. ${ }^{42,43}$ Nesse sentido, no presente trabalho, o modelo comumente utilizado da ADMPC (Figura 3(A)) foi reduzido a um esqueleto de amilose ligado a apenas uma cadeira lateral do grupo tris-(3,5-dimetilfenilcarbamato) (Figura 3(C)), a fim de reduzir o custo computacional e o tempo de cálculo atrelado ao sistema supramolecular. Cabe ressaltar que esse procedimento metodológico já foi feito com sucesso em trabalhos anteriores do nosso grupo de pesquisa, envolvendo separações enantioméricas. ${ }^{32,33}$

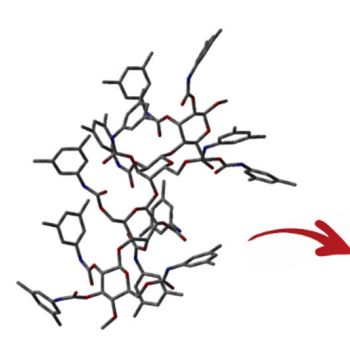

(A)

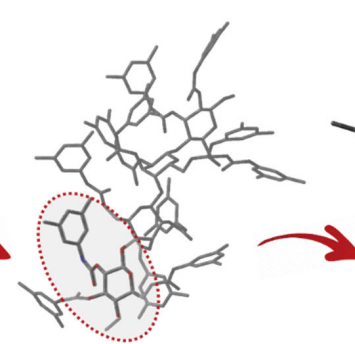

(B)

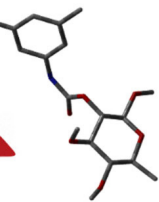

(C)
Figura 3. Em (A) modelo completo de estrutura helicoidal, comumente usado em simulações de dinâmica e docking molecular, ${ }^{27-29} \mathrm{em}(\boldsymbol{B})$ parte do modelo completo original, evidenciando o grupo tris-(3,5-dimetilfenilcarbamto) $e$ em $(\boldsymbol{C})$ modelo original reduzido a um esqueleto de amilose ligado a uma cadeira lateral do grupo tris-(3,5-dimetilfenilcarbamato) na qual haverá interação com os enantiômeros do analito, usado em trabalhos anteriores do nosso grupo de pesquisa ${ }^{32,33}$

Uma vez obtidas as estruturas otimizadas, em nível M06-2X/6-31G(d,p), para os enantiômeros da OXI, bem como para o modelo reduzido da ADMPC (MR-ADMPC) e levando-se em conta os valores numéricos de densidade de carga sob cada átomo em destaque nos mapas de potencial eletrostático molecular (MPE) para as espécies interagentes (Figura 4), foi proposta a formação de duas ligações de hidrogênio, a fim de maximizar a interação entre a OXI e o MR-ADMPC: (i) a primeira ligação (LIGAÇÃO A), considerou-se a interação entre um átomo de hidrogênio (H1) ligado a um átomo de oxigênio $(\mathrm{O} 2)$ do analito e um átomo de oxigênio (O1') da MR-ADMPC; (ii) já a segunda ligação (LIGAÇÃO B) levou em conta a interação entre átomo de oxigênio (O1) da OXI, e um átomo de hidrogênio (H1') ligado a um átomo de nitrogênio (N1') da fase estacionária (ver numeração dos átomos supracitados na Figura 4).

Considerando as duas ligações (A e B) propostas, foram obtidos dois CDTs distintos, os quais foram nomeados $(R)$-OXI... [MR-ADMPC] e (S)-OXI...[MR-ADMPC]. A Tabela 1 mostra as propriedades energéticas, $\Delta \mathrm{E}$ e $\Delta \mathrm{G}$, calculadas para os respectivos 
Tabela 1. Valores de $\Delta \mathrm{E}$ e $\Delta \mathrm{G}$ calculados para os CDTs, em meio de n-hexano, no nível M06-2X/6-31G(d,p)

\begin{tabular}{|c|c|c|c|c|}
\hline CDT & $\Delta \mathrm{E}$ & $\Delta \Delta \mathrm{E}$ & $\Delta \mathrm{G}$ & $\Delta \Delta \mathrm{G}$ \\
\hline$(R)-\mathrm{OXI} \ldots[\mathrm{MR}-\mathrm{ADMPC}]$ & $-14,9(-11,2)$ & $2,6(2,1)$ & $2,4(3,8)$ & $6,6(3,7)$ \\
\hline (S)-OXI...[MR-ADMPC] & $-17,5(-13,3)$ & & $-4,2(0,1)$ & \\
\hline
\end{tabular}

${ }^{\mathrm{a}}$ Entre parênteses estão as energias de complexação corrigidas pelo BSSE. Valores em kcal mol ${ }^{-1}$.

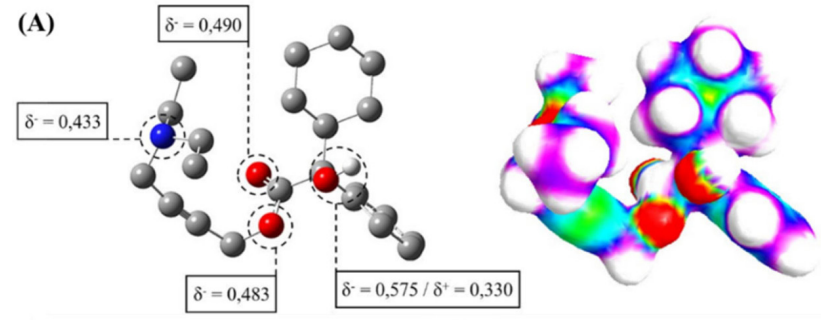

(B)

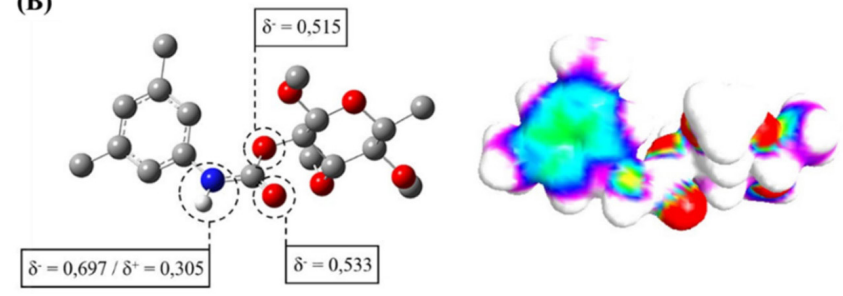

Figura 4. Mapas do Potencial Eletrostático obtidos para as estruturas otimizadas da: (A) OXI e (B) MR-ADMPC, com destaque para as densidades de carga sob os átomos considerados sítios de interação. Os átomos de hidrogênio foram omitidos para facilitar a visualização

CDTs, cujos valores foram estimados em meio contendo $n$-hexano, através do modelo de solvatação implícito SMD.

Analisando os dados dispostos na Tabela 1, é possível observar, a partir dos valores de $\Delta \mathrm{E}$ e $\Delta \mathrm{G}$, que o complexo (S)-OXI...[MRADMPC] é ligeiramente mais estável que o complexo ( $R$ )-OXI... [MR-ADMPC]. Este fato pode explicado através das interações intermoleculares estabelecidas em cada CDT (Figura 5).

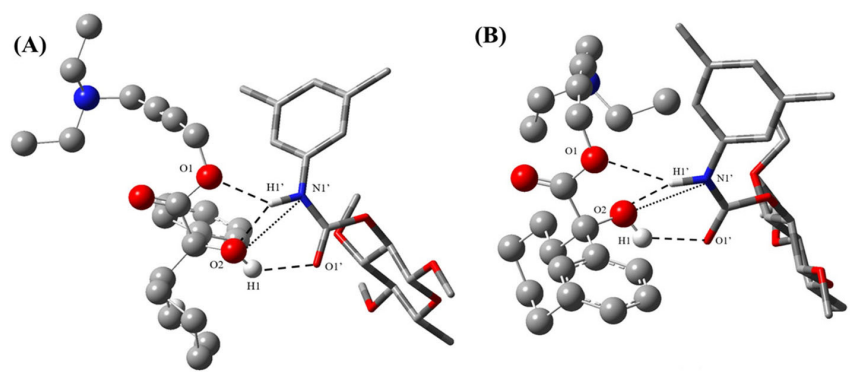

Figura 5. Geometrias otimizadas dos CDTs: (A) (R)-OXI...[MR-ADMPC] e (B) (S)-OXI... [MR-ADMPC], evidenciando as ligações de hidrogênio e interações $N$ '-O estabelecidas, as quais são representadas por linhas tracejadas e pontilhadas, respectivamente. Os átomos de hidrogênio não envolvidos nas interações foram omitidos para facilitar a visualização

Apesar de ambos CDTs apresentarem o mesmo tipo e mesmo número de interações intermoleculares, as ligações de hidrogênio formadas a partir dos átomos O2, O1 e H1 da OXI, assim como, a interação dipolar O2-N1', são essenciais para uma melhor compreensão da maior estabilidade do complexo $(S)$-OXI...[MR-ADMPC] em relação ao (R)-OXI...[MR-ADMPC]. Nota-se claramente na Tabela 2 que as distâncias de interação são sempre menores para o complexo $(S)$-OXI...[MR-ADMPC] em comparação ao (R)-OXI...[MR-ADMPC], com destaque para a interação O1-H1', cerca de 0,4 Å menor, tornando-a o principal responsável pela diferença de energia entre os complexos. Desse modo, evidencia-se que as interações mais curtas, portanto, mais fortes do ponto de vista eletrostático, acabam promovendo, uma sutil maior estabilidade para o complexo (S)-OXI...[MR-ADMPC]. Além disso, como pode ser visto na Tabela 1 (valores entre parênteses), a estabilidade relativa entre os complexos não muda quando o BSSE foi considerado.

Devido à semelhança estrutural dos CDTs, era de se esperar que a diferença energética entre eles fosse, de fato, pequena. Apesar disso, a ligeira diferenciação energética, bem como na magnitude das forças de interações estabelecidas, sugere, em nível molecular, a discriminação quiral.

Tabela 2. Comprimentos das interações intermoleculares estabelecidas nos CDTs

\begin{tabular}{ccccc}
\hline CDT & O1-H1' & O2-H1' & H1-O1' & O2-N1' \\
\hline$(R)$-OXI-ADMPC & $2,62 \AA$ & $1,98 \AA$ & $2,04 \AA$ & $2,87 \AA$ \\
$(S)$-OXI-ADMPC & $2,21 \AA$ & $1,94 \AA$ & $1,97 \AA$ & $2,81 \AA$ \\
\hline
\end{tabular}

Finalmente, considerando os resultados energéticos e estruturais obtidos, verifica-se que a OEE corrobora com os resultados previstos experimentalmente, com o uso da fase estacionária ADMPC e $n$-hexano como fase móvel. Assim, conclui-se que $(R)$-OXI eluirá primeiro do que o $(S)$-OXI, uma vez que o complexo (R)-OXI...[MR-ADMPC] é menos estável e, portanto, com menor retenção no sistema cromatográfico.

\section{CONCLUSÕES}

No presente trabalho realizou-se um estudo teórico/experimental com o intuito de entender o processo de separação enantiosseletiva, bem como a ordem de eluição do fármaco antimuscarínico OXI, o qual tem sido amplamente utilizado em doenças do trato urinário. Na primeira etapa, a separação enantiosseletiva da OXI foi avaliada via HPLC, empregando-se a coluna Chiralpak ${ }^{\circledR}$ AD. Após vários testes, variando-se as proporções da fase móvel e solventes orgânicos modificadores, constatou-se que $n$-hexano: isopropranol: etanol: dietilamina (94: 4: 2: 0,05, v/v/v/v) foi suficiente para promover uma separação de linha de base (Resolução, $R s>3$ ) dos enantiômeros. Assim, a ordem de eluição enantiomérica foi conseguida, evidenciando no sistema cromatográfico que $(R)$-enantiômero do fármaco elui mais rapidamente que seu antípoda $(S)$-enantiômero.

Na segunda etapa, a fim de compreender o processo de separação enantiosseletiva da OXI, em nível molecular, cálculos DFT foram realizados, em nível M06-2X/6-31G(d,p). De acordo com os resultados obtidos, foi possível entender a natureza das interações envolvidas no processo de separação, por meio da obtenção das estruturas otimizadas, em meio de $n$-hexano, dos CDTs formados entre os enantiômeros da OXI e o modelo reduzido da fase estacionária. As estabilidades dos complexos, analisadas através dos valores de $\Delta \mathrm{E}$ e $\Delta \mathrm{G}$, foram explicadas pela formação de ligações de hidrogênio estabelecidas. Além disso, o estudo teórico contribuiu 
para explicação, em nível molecular, da ordem de eluição obtida experimentalmente. Por fim, o presente trabalhou mostrou como a Química Computacional pode ser extremamente útil na elucidação do reconhecimento quiral em separações enantiosseletivas.

\section{AGRADECIMENTOS}

Os autores agradecem à Fundação de Amparo à Pesquisa do Estado de Minas Gerais (FAPEMIG), ao Conselho Nacional de Desenvolvimento Científico e Tecnológico $(\mathrm{CNPq})$ e à Coordenação de Aperfeiçoamento de Pessoal de Nível Superior - Brasil (CAPES, Finance Code 001) pelo suporte financeiro. Este trabalho é resultado também da colaboração científica entre pesquisadores membros da Rede Mineira de Química (RQ-MG), cujo projeto é financiado pela FAPEMIG.

\section{REFERÊNCIAS}

1. Hsu, F. C.; Weeks, C. E.; Selph, S. S.; Blazina, I.; Holmes, R. S.; McDonagh, M. S.; Int. Urogynecol. J. 2019, 30, 1603.

2. Whalen, K.; Finkel, R.; Panavelil, T. A.; Farmacologia Ilustrada. $6^{\text {a }}$ ed. Artmed: Porto Alegre, 2016.

3. Abrams, P.; Andersson, K.; BJU Int. 2007, 100, 897.

4. Guay, D. R. P.; Clin. Pharmacokinet. 2003, 42, 1243.

5. Kruse, A. C.; Kobilka, B. K.; Gautam, D.; Sexton, P. M.; Christopoulos, A.; Wess, J.; Nat. Rev. Drug Discov. 2014, 13, 549.

6. McCrery, R. J.; Appell, R. A.; Ther. Clin. Risk Manage. 2006, $2,19$.

7. Walters, K. A.; Lane, M. E. Em Dermal Drug Delivery: From Innovation to Production; Ghosh, T. K., eds.; CRC Press: Boca Raton, 2020.

8. Noronha-Blob, L.; Kachur, J. F.; J. Pharmacol. Exp. Ther. 1991, 256, 562 .

9. Arisco, A. M.; Brantly, E. K.; Kraus, S. R.; Drug Des., Dev. Ther. 2009 , 3,151 .

10. Dmochowski, R. R.; Starkman, J. S.; Davila, G. W.; Int. Braz. J. Urol. 2006, 32, 513 .

11. Kennelly, M. J.; Rev. Urol. 2010, 12, 12.

12. Smith, E. R.; Wright, S. E.; Aberg, G.; Fang, Y.; McCullough, J. R.; Arzneimittelforschung 1998, 48, 1012.

13. Sathyan, G.; Chancellor, M. B.; Gupta, S. K.; Br. J. Clin. Pharmacol. 2001, 52, 409 .

14. Mizushima, H.; Takanaka, K.; Fukazawa, I.; Ishizuka, H.; Xenobiotica 2007, 37, 59

15. Zobrist, R. H.; Schmid, B.; Feick, A.; Quan, D.; Sanders, S. W.; Pharm. Res. 2001, 18, 1029.

16. Staskin, D. R.; Traub, S.; Curr. Bladder Dysfunct. Rep. 2009, 4, 34.

17. Zhang, Y.; Wu, D. R.; Wang-Iverson, D. B.; Tymiak, A. A.; Drug Discov. Today. 2005, 10, 571.

18. Bonato, P. S.; Jabor, V. A. P.; Gaitani, C. M.; Quim. Nova. 2005, 28, 683.

19. Gübitz, G.; Schmid, M. G.; Biopharm. Drug Dispos. 2001, 22, 291.

20. Scriba, G. K. E.; J. Chromatogr. A 2016, 1467, 56.

21. Berthod, A.; Anal. Chem. 2006, 78, 2093.

22. Scriba, G. K. E.; Chromatographia. 2012, 75, 815.
23. Teixeira, J.; Tiritan, M. E.; Pinto, M. M. M.; Fernandes, C.; Molecules. 2019, 24, 865 .

24. Chankvetadze, B.; TrAC - Trends Anal. Chem. 2019, 122, 115709.

25. Alebic-Kolbah, T.; Zavitsanos, A. P.; J. Cromatogr. A 1997, 759, 65.

26. Da Fonseca, P.; De Freitas, L. A. P.; Pinto, L. F. R.; Pestana, C. R.; Bonato, P. S.; J. Chromatogr. B. 2008, 875, 161.

27. Liu, Y.; Cai, L.; Lun, J.; Zhao, M.; Guo, X.; New J. Chem. 2020, 42, 18337.

28. Zhu, B.; Zhao, F.; Yu, J.; Wang, Z.; Song, Y.; Li, Q.; New J. Chem. 2018, 16,13421

29. Zhao, Y.; Li, S.; Wang, X.; Yu, J.; Song, Y.; Guo, X.; Chirality 2019, 31, 502.

30. Kasat, R. B.; Wang, N. H. L.; Franses, E. I.; J. Chromatogr. A 2008, 1190,110

31. Del Rio, A.; Hayes, J. M.; Stein, M.; Piras, P.; Roussel, C.; Chirality 2004, 16, S1.

32. Silva, C. F.; Guimarães, L.; Borges, K. B.; Nascimento Jr., C. S.; Chirality 2019, 32, 53.

33. Silva, C. F.; Do Nascimento, T. A.; Guimarães, L.; Borges, K. B.; Nascimento Jr., C. S.; Chirality 2020, 32, 353.

34. Hohenstein, E. G.; Chill, S. T.; Sherrill, C. D.; J. Chem. Theory Comput. 2008, 4, 1996.

35. Zhao, Y.; Truhlar, D. G.; Acc. Chem. Res. 2008, 41, 157.

36. Marenich, A. V.; Cramer, C. J.; Truhlar, D. G.; J. Phys. Chem. B 2009, 7, 6378 .

37. Surján, P.R.; Császár, P.; Poirier, R. A.; Van Lenthe, J. H.; Acta Phys. Hung. 1990, 67, 387.

38. Boys, S. F.; Bernardi, F.; Mol. Phys. 1970, 19, 553.

39. Frisch, M. J.; Trucks, G. W.; Schlegel, H. B.; Scuseria, G. E.; Robb, M. A.; Cheeseman, J. R.; Scalmani, G.; Barone, V.; Mennucci, B.; Petersson, G. A.; Nakatsuji, H.; Caricato, M.; Li, X.; Hratchian, H. P.; Izmaylov, A. F.; Bloino, J.; Zheng, G.; Sonnenberg, J. L.; Hada, M.; Ehara, M.; Toyota, K.; Fukuda, R.; Hasegawa, J.; Ishida, M.; Nakajima, T.; Honda, Y.; Kitao, O.; Nakai, H.; Vreven, T.; Montgomery, J. A., Jr.; Peralta, J. E.; Ogliaro, F.; Bearpark, M.; Heyd, J. J.; Brothers, E.; Kudin, K. N.; Staroverov, V. N.; Kobayashi, R.; Normand, J.; Raghavachari, K.; Rendell, A.; Burant, J. C.; Iyengar, S. S.; Tomasi, J.; Cossi, M.; Rega, N.; Millam, J. M.; Klene, M.; Knox, J. E.; Cross, J. B.; Bakken, V.; Adamo, C.; Jaramillo, J.; Gomperts, R.; Stratmann, R. E.; Yazyev, O.; Austin, A. J.; Cammi, R.; Pomelli, C.; Ochterski, J. W.; Martin, R. L.; Morokuma, K.; Zakrzewski, V. G.; Voth, G. A.; Salvador, P.; Dannenberg, J. J.; Dapprich, S.; Daniels, A. D.; Farkas, Ö.; Foresman, J. B.; Ortiz, J. V.; Cioslowski, J.; Fox, D. J.; Gaussian 09, revision A.1, Gaussian Inc., Wallingford CT, 2009.

40. Ali, I.; Al-Othman, Z. A.; Al-Warthan, A.; Alam, S. D.; Farooqi, J. A.; Chirality 2014, 26, 136.

41. Zhang, P.; Sun, G.; Tang, K.; Yang, W.; Sui, G.; Zhou, C.; J. Sep. Sci. 2014, 37, 3443 .

42. Okamoto, Y.; Kaida, Y.; J. Chromatogr. A 1995, 666, 403.

43. Zhou, Y.; Ma, C.; Wang, Y.; Zhang, Q. M.; Zhang, Y. Y.; Fu, J.; Gao, H. Zhao, L. X.; J. Pharm. Anal. 2012, 2, 48. 